\title{
Application of 3D Ray Tracer in WiMAX
}

\author{
Wang Chao \\ Zhengzhou Electromechanical Engineering Research Institute, China \\ 854909839@qq.com
}

Keywords: Wimax; 3D Ray Tracer; Carrier Aggregation; Inter-Cell Interference (ICI); System Level Performance.

\begin{abstract}
WiMAX is a promising technology for a higher spectral efficiency and reliable transmission. This paper compares the downlink throughput experienced by mobile users in a macro-cell in an interference limited scenario with a frequency reuse of one. Bit accurate link level simulations are performed for the downlink physical shared channel (PDSCH). The study is performed for a single antenna system in specific routes at two different carrier frequencies $(800 \mathrm{MHz}$ and 2.6 $\mathrm{GHz}$ ), for two urban environments in the China, and two cell sizes. The paper also considers the carrier aggregation technique which has been proposed since it is difficult to ensure a single wide frequency band for operators. A state-of-the-art 3D ray tracer tool is used to generate 3D channel models for accurate modelling of channel statistics. The study shows that the $800 \mathrm{MHz}$ band has no greater effect on the downlink throughput as compared to $2.6 \mathrm{GHz}$ band, which leads to no additional advantages of inter-band aggregation (considering $800 \mathrm{MHz}$ ) compared to intra-band aggregation. It is also observed that a range of speeds can be handled while maintaining good performance in WiMAX. We have clearly shown that the choice of cell size by mobile operators for optimum coverage depends mainly on the propagation characteristic of the environment.
\end{abstract}

\section{Introduction}

WiMAX is one of the $4 \mathrm{G}$ wireless cellular communication standards which is designed to operate in evolved universal terrestrial radio access (E-UTRA) frequency bands in the range of 699 $\mathrm{MHz}$ to $3.8 \mathrm{GHz}$ [1]. E-UTRA low frequency operating bands 5, 8, 12, 13, 14, 17, 18, 19, 20, are considered attractive bands due to their enhanced propagation characteristics. In order to meet the increasing demand for mobile communication services with increasing number of users, the IEEE WiMAX system has proposed a set of promising technologies, e.g., enhanced MIMO and carrier aggregation (CA) that can provide higher spectral efficiency and reliable transmission [2]. CA has been considered as an important technology, where more than one component carriers (CC) are aggregated to support higher data rates. CA of up to five times the standard LTE bandwidth is supported in WiMAX to achieve a maximum downlink (DL) data rate of 1 Gbps and uplink (UL) data rate of 500 Mbps. CA_7-20 denotes inter-band carrier aggregation between EUTRA bands 7 and 20 for UL and DL as planned by the IEEE technical specification group for Release 11 of the LTE standard [1]. The selection of $800 \mathrm{MHz}$ and $2.6 \mathrm{GHz}$ in this study is based on the DL frequency range of bands 20 and 7 respectively. While standardization of CA has progressed a small number of studies for system level performance of various CA scenarios have been made. Some of these studies have claimed that CA provides a powerful means to boost the peak user throughput in WiMAX to meet the IMT-Advanced requirements set by the ITU-R. However these studies have not considered interference when evaluating CA performance, while others did not consider accurate modelling of the wireless channels [2]-[5]. Our study considers site specific deterministic channel modelling for accurate analysis of CA performance considering different cell sizes.

This evaluation of WiMAX downlink throughput will also consider interference from adjacent cells. Inter-cell interference (ICI) is inevitable in such systems and will degrade the UE performance especially for users at the cell edge. WiMAX systems are expected to achieve the goal of frequency reuse of one or nearly close to one in practice [6]. Here we study the performance 
of WiMAX PDSCH for a user moving toward the cell edge considering the interference from all adjacent cells. Column widths, line spacing, and type styles are built-in; examples of the type styles are provided throughout this document and are identified in italic type, within parentheses, following the example. PLEASE DO NOT RE-ADJUST THESE MARGINS. Some components, such as multi-leveled equations, graphics, and tables are not prescribed, although the various table text styles are provided. The formatter will need to create these components, incorporating the applicable criteria that follow.

\section{Channel Models and Deployment Scenarios}

The channels used in this paper are generated using a ray tracing tool integrated with measured BS and UE antenna patterns. The ray tracing analysis is based on point-to-point predictions from each BS to every UE location. The propagation channel in the ray tracer is modelled as a set of spatial and temporal multipath components, where the ray model provides information on the amplitude, phase, time delay, angle of arrival (AoA) and angle of departure (AoD) in elevation and azimuth planes. The ray tracing engine identifies all possible ray paths between the BS and UE based on an urban site specific database, given that the database includes terrain, buildings and foliage related information. A validation study of the ray tracer was presented in [9]. The $3 \mathrm{D}$ statistics provided by this tracer will result in more accurate modelling as the assumption of 2D propagation May results in inaccurate estimation of system level performance [10].

The system was modelled using measured BS and UE antenna patterns. The radiation patterns of the macro-cell BS antenna and the UE antenna used in this study were measured in an anechoic chamber at the University of Zhengzhou. All patterns are 3D and include full phase and polarization information. The total power radiation patterns are shown in Fig. 1.

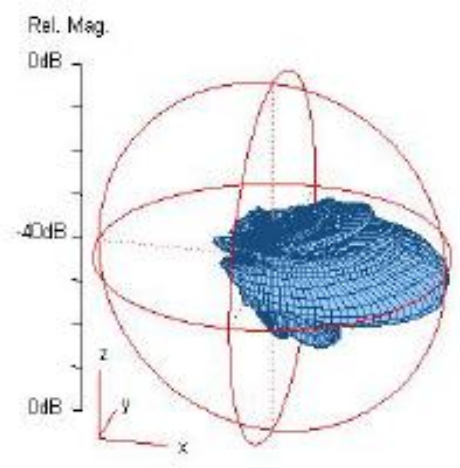

(a) Macro BS antenna

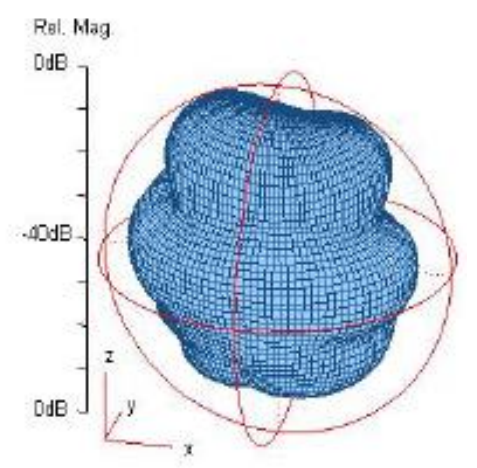

(b) UE handset antenna

Figure 1. Total power measured radiation patterns

\section{Analysis of Throughput Results}

In this paper, the system level simulation combining ICI modelling with a detailed WiMAX PDSCH simulator is implemented. The simulator is used to evaluate the performance of each supported modulation and coding scheme (MCS). The MCS mode that maximises the link-level throughput is chosen by the link adaptation algorithm. The results were obtained by considering a UE moving with a certain speed in specific routes.

Let us first compare the performance of the PDSCH in an interference limited with an interference free scenario. Fig. 2 shows the differences between the two scenarios in Zhengzhou at a speed of $17 \mathrm{~m} / \mathrm{s}$ and the effect interference has on the system throughput, SINR levels, and variance of the estimation error. The comparison has been carried out for two frequency bands $(800 \mathrm{MHz}$ and $2.6 \mathrm{GHz})$. The results show that in the interference free case, the estimated throughput is always high even when the user approaches the cell edge which is due to the high SINR levels experienced by the UE along the route. It is clear from Fig. 2c and Fig. 2d that when ICI is considered, the SINR levels are lower by approximately $40 \mathrm{~dB}$ and $30 \mathrm{~dB}$ for $800 \mathrm{MHz}$ and $2.6 \mathrm{GHz}$ bands respectively. Such differences in the 
SINR level lead to lower estimated throughput experienced by UEs when interference is considered. Fig. 4e and Fig. 4f show that the reduction in the SINR levels caused by the ICI will increase the variance of the estimation error (and the estimation error). For routes with low SINR levels, the performance of the channel estimator is expected to be worse. Hence the system level analysis of LTE-Advanced PDSCH will lead to overestimation of system performance in terms of physical throughput.

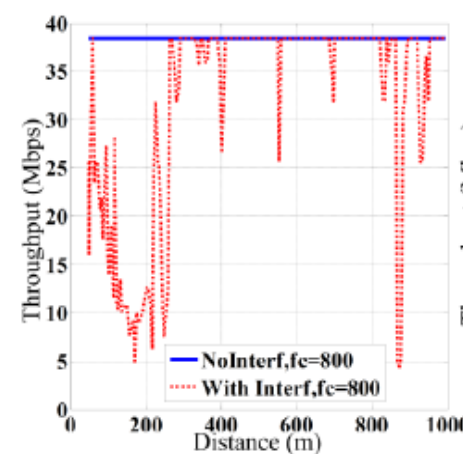

(a) Throughput at $800 \mathrm{MHz}$

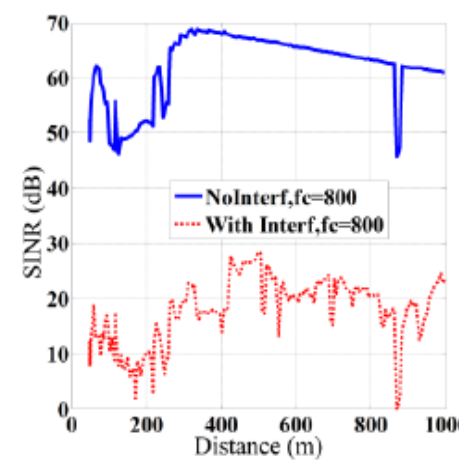

(c) SINR at $800 \mathrm{MHz}$

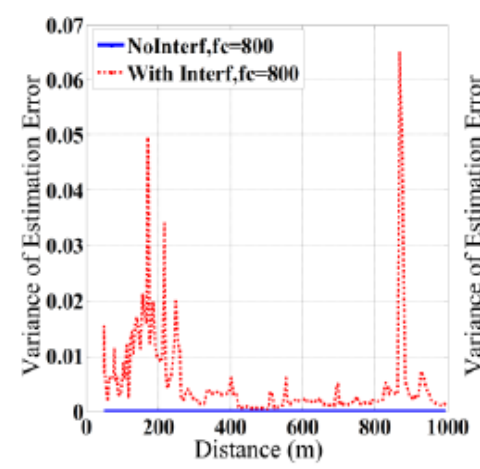

(e) Variance estim. error at $800 \mathrm{MHz}$

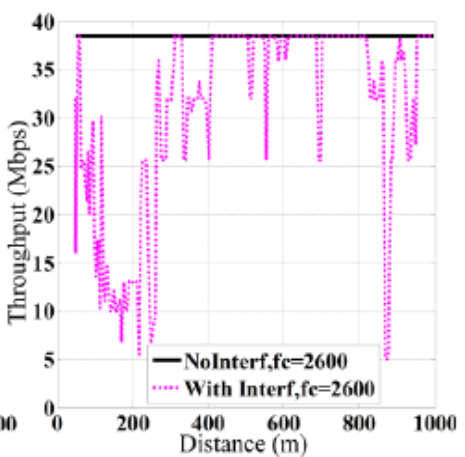

(b) Throughput at $2.6 \mathrm{GHz}$

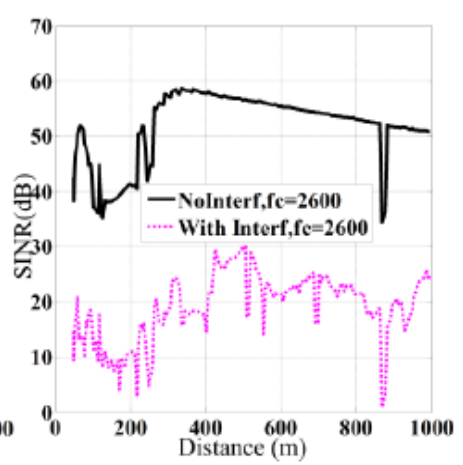

(d) SINR at $2.6 \mathrm{GHz}$

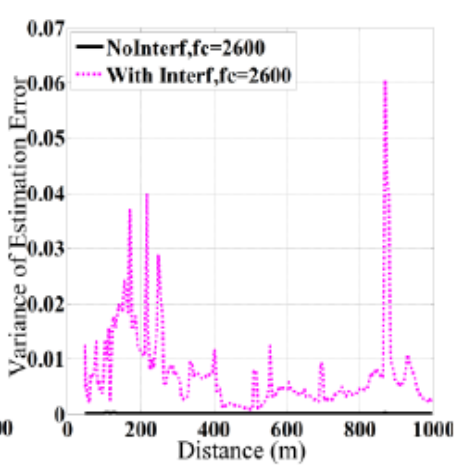

(f) Variance estim. error at $2.6 \mathrm{GHz}$

Figure 2. Comparison of WiMAX downlink performance with and without interference at $800 \mathrm{MHz}$ and $2.6 \mathrm{GHz}$ in Zhengzhou at speed of $17 \mathrm{~m} / \mathrm{s}$

Let us now compare the performance of WiMAX PDSCH in case of inter and intra band carrier aggregation. Fig. 3 shows the throughput of intra-band CA at $800 \mathrm{MHz}$, intra-band CA at $2.6 \mathrm{GHz}$ to achieve a contiguous aggregated bandwidth of $20 \mathrm{MHz}$. The $\mathrm{CA}$ is also performed for inter-band CA across the $800 \mathrm{MHz}$ and $2.6 \mathrm{GHz}$. The ICI is also considered in this analysis and the results clearly show that inter-band $\mathrm{CA}$ by using the $800 \mathrm{MHz}$ does not offer higher throughput as expected in literature (due to the good propagation characteristic of $800 \mathrm{MHz}$ band). This is due to the higher interference experienced at $800 \mathrm{MHz}$. The consideration of interference results in very close SINR levels experienced by $\mathrm{UE}$ at the $800 \mathrm{MHz}$ and $2.6 \mathrm{GHz}$ bands as illustrated in Fig. 2c and Fig. 2d. This consequently leads to no additional advantages of inter- band CA (considering $800 \mathrm{MHz}$ ) compared to contiguous intra-band CA. 


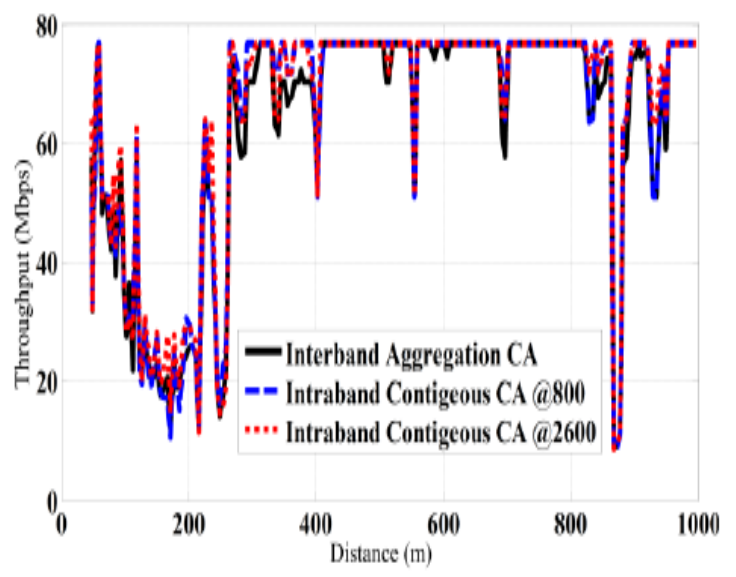

Figure 3. Throughput in inter and intra band CA with interference
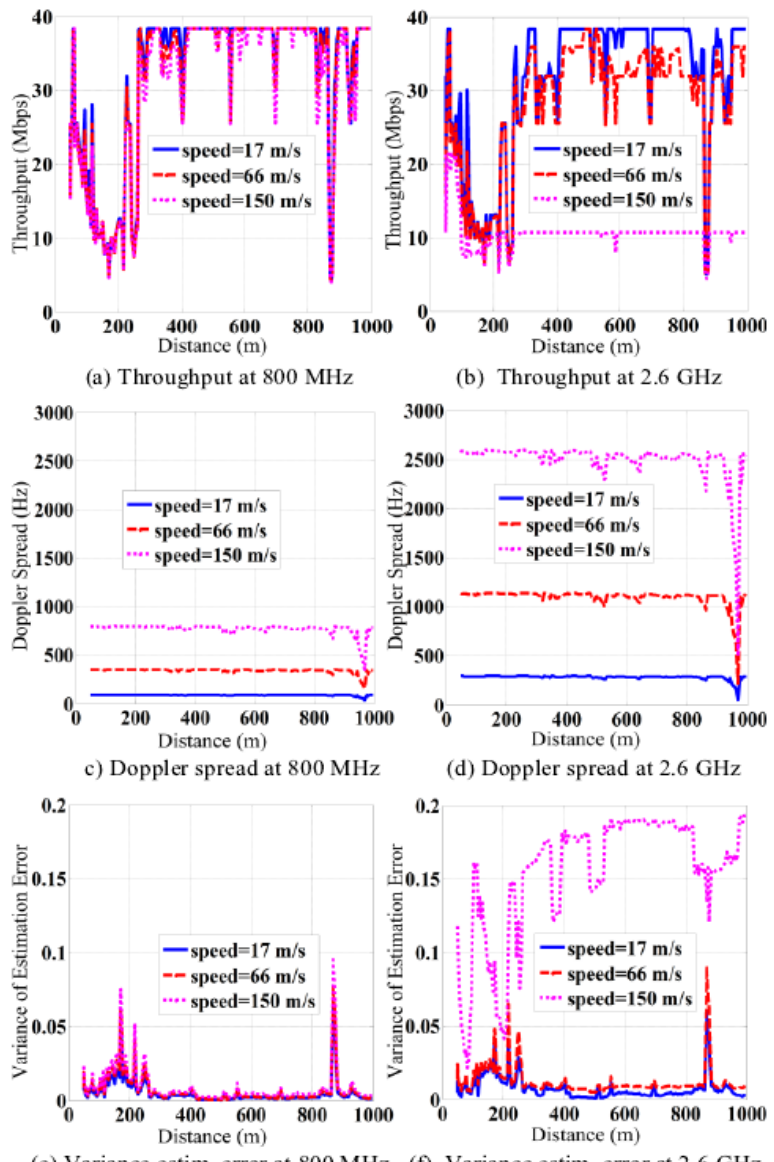

(e) Variance estim. error at $800 \mathrm{MHz}$ (f) Variance estim. error at $2.6 \mathrm{GHz}$

Figure 4. Comparison of LTE-Advanced downlink performance with interference at $800 \mathrm{MHz}$ and 2.6 $\mathrm{GHz}$ in London and different speeds.

Fig. 4 shows a comparison of the performance between the $800 \mathrm{MHz}$ and $2.6 \mathrm{GHz}$ bands for the bandwidth of $10 \mathrm{MHz}$ in Zhengzhou. The analysis is performed for three different mobility speeds 17 $\mathrm{m} / \mathrm{s}, 66 \mathrm{~m} / \mathrm{s}$, and $150 \mathrm{~m} / \mathrm{s}$ which are applicable to car and train mobility. Fig. $6 \mathrm{c}$ and Fig. $6 \mathrm{~d}$ show the Doppler spread for differen speeds for the $800 \mathrm{MHz}$ and $2.6 \mathrm{GHz}$ bands respectively.

The Doppler spread caused by the $800 \mathrm{MHz}$ is lower compared to the $2.6 \mathrm{GHz}$, where the speed of $150 \mathrm{~m} / \mathrm{s}$ cause a maximum Doppler spread of around $800 \mathrm{~Hz}$, while in case of $2.6 \mathrm{GHz}$, the Doppler spread is around $2.5 \mathrm{kHz}$. The throughput obtained at different speeds is illustrated in Fig. $4 \mathrm{a}$ and Fig. 4b. In the case of the $800 \mathrm{MHz}$ band, the three speeds result in close performance since the coherence time is still larger than the OFDM symbol duration of WiMAX system. Therefore the WiMAX system is offering good performance in the range of speeds considered. 
In the $2.6 \mathrm{GHz}$ band, it is shown that the increase in speed from $17 \mathrm{~m} / \mathrm{s}$ to $66 \mathrm{~m} / \mathrm{s}$ will slightly degrade the performance, however, the throughput at $150 \mathrm{~m} / \mathrm{s}$ drops from $40 \mathrm{Mbps}$ to $10 \mathrm{Mbps}$ and remains at this rate for the rest of the locations in the route. This is due to the higher Doppler spread in the $2.6 \mathrm{GHz}$ and the higher variance of estimation error at $150 \mathrm{~m} / \mathrm{s}$ as shown in Fig. $4 \mathrm{f}$ compared to Fig. 4e for $800 \mathrm{MHz}$. We conclude that the WiMAX is capable of handling high speed (up to speed range considered) while maintaining good performance.

\section{Conclusion}

In this paper we have presented a quantitative analysis of the WiMAX PDSCH in terms of throughput and other performance related parameters such as the estimation error statistics, Doppler spread, and channel related statistics. The analysis compared the performance of the PDSCH in case of interference. The results obtained show that when interference is not considered, the system level analysis of WiMAX PDSCH will lead to overestimation of system performance.

The study has also looked at the potential gain of inter-band CA as compared to intra-band aggregation. We have shown that no additional advantage of inter-band aggregation (considering 800 $\mathrm{MHz}$ ) is gained in comparison to intra-band aggregation. The analysis also clearly showed that WiMAX is capable of handling high speed (up to range considered) with quite good performance. Finally we have considered the performance differences in case of different cell radiuses. We observed that the selection of the cell size by mobile operators depends mainly on the propagation characteristic of the environment.

\section{References}

[1] 3GPP TS 36.101: Evolved Universal Terrestrial Radio Access (E-UTRA): User Equipment (UE) radio transmission and reception, V10.10.0, March 2013.

[2] C. Park, H. Jung, and S. Kim, System Level Performance Evaluation of Various Carrier Aggregation Scenarios in WiMAX Communication, 15th IEEE International Conference on Advanced Computing Technologies, September 2013.

[3] M. Iwamura, K. Mo-Han, R. Nory, Carrier Aggregation Framework in 3GPP WiMAX , IEEE Communication Magazine, vol.48, no.8, August 2012,pp.60-97.

[4] M.Abduljawad, M.Al-Shibly, M. Habaebi, Carrier Aggregation in Long Term EvloutionAdvanced, 3rd IEEE Control and System Graduate Research Colloquium, July 2012.

[5] G. Yuan, X. Zhang, W. Wang, Carrier Aggregation for WiMAX Mobile Communication Systems , IEEE communication magazine ,vol.48, no.2, February 2012, pp.88-93.

[6] H. Sari, S. Sezginer, E.Vivier, Full frequency reuse in mobile WiMax and LTE networks with sectored cells, 3rd IEEE Mobile WiMax Symposium, July 2009.

[7] 3GPP TS 36.211: Evolved Universal Terrestrial Radio Access (E-UTRA): Physical Channels and Modulation, V10.4.0, December 2011.

[8] 3GPP TS 36.212: Evolved Universal Terrestrial Radio Access (E-UTRA): Multiplexing and channel coding, V10.5.0, March 2012.

[9] E.Tameh, A. Nix, The Use of Measurement Data to Analyse the Performance of Rooftop Diffraction and Foliage Loss Algorithms in a 3-D Integrated Urban/Rural Propagation Model, 48th IEEE Vehicular Technology Conference, May 1998.

[10] R. Almesaeed, A. Ameen, A. Doufexi, et al, A Comparison Study of 2D and 3D ITU Channel Model, 6th IEEE Wireless Days, November 2013.

[11] M. Bugeja, Physical Layer Performance of LTE System with Multiple Anetnnas, Ph.D. dissertation, Dept. Elect. Eng., Bristol Univ., Bristol, 2009.

[12] A. Ameen, E. Mellios, A. Doufexi, et al,WiMAX Downlink Throughput Evaluation in the 3G and TV White Space Bands, 24th IEEE International Symposium on Personal, Indoor and Mobile Radio Communications, September 2013. 